\title{
ENERGY EFFICIENT ROUTING PROTOCOL FOR MOBILE SOCIAL SENSING NETWORKS
}

\author{
SALEM SATI, AHMED SOHOUB, AND TAREG ABULIFA*
}

\begin{abstract}
Mobile Social Sensing Network (MSSN) is a subclass of Wireless Sensor Networks (WSN). This MSSN is consists of mobile sensing transducers carried by people. Sensing information gathered by mobile sensors will transmit to the data sink. This data sink may it is fixed or mobile. But in optimal cases, it should have efficient energy and position compared with other mobile sensors. On the other hand, mobile sensors may have a social tie because they carried by people. Traditional MANET routing protocols such as AODV and DSR are inapplicable or perform poorly for mobile social data sensing. Especially for distributed mobile social sensing. Insufficient performance due to the nature of the mobile sensors which suffering from a limited energy source. In recent days, there are many routing protocols proposed by researchers. These protocols improve the total delivered messages in mobile social sensing networks, but most of them do not take into account the link bandwidth and node storage limitation, thus routing may lead to more energy consumption among mobile sensing nodes. In this paper, we design an Energy-Efficient Routing Protocol (EERP) for mobile social sensing networks. We consider the node energy as a balance function between the delay of collected data and transmission of sensor nodes to the data sink. Furthermore, we also develop an enhanced version of the suggested EERP which named EERP+S, EERP+S combines the energy percentage and social metric of node degree. EERP and its updated version EERP $+\mathrm{S}$ are dynamically adjusting the control function based on data delay and transmission in addition to node activity. Simulation results demonstrate the efficiency of EERP and EERP+S compared with the flooding behavior of an Epidemic. Epidemic and its social version Ep-Soc are compared with suggested protocols in distributed mobile social sensing paradigms.
\end{abstract}

Key words: Energy Efficiency, Mobile Sensing, Social Sensing, Node Energy, Routing Performance, Forwarding Decision

AMS subject classifications. 68M12

1. Introduction. Mobile Social Sensor Network (MSSN) is a future network with a higher increase in social sensing application scenarios. Due to recent advanced technology in smartphones carried by people, it is easily feasible for the mobile and fixed sensor not only to collect industrial environment sensed data, but also to gather data in social and environment of real-time applications.

There are a lot of mobile sensing applications with different sensing functions. Where the nodes maybe humans, cars or animals which may be connected to the internet. Different sensing mobile nodes collecting data for applications that form a new sensing topology paradigm. Socially scenarios based on smartphones and other sensing scenarios based on car or animal tracking are applications of mobile sensing $[1,2,3]$. Those scenarios are collecting and processing sensing data from different mobile sensors nodes. The data collecting is one of the challenges on the performance of mobile sensing. This challenge depends on how to efficiently gathering the sensing data with the limitation of bandwidth and storage. One possible solution is to deploy mobile devices to transmit sensing data rather than deploy a traditional fixed data sink.

Mobile sensors nodes are energy-constrained devices. Therefore, MSSN researchers from different parts of the WSN group are trying to minimize energy consumption. This energy prevention is for increasing the nodes' lifetime on the network. In real applications of MSSN, nodes lifetime should be longer as it is possible. This mobile sensor activity of sensing and transmission must be without communication interruption. Specially interruption between mobile sensors and head cluster (data sink).

There are MSSN applications that should be reported to the data sink on time. This is because the application sensing real-time which sent within a shorter time. Besides, the system monitoring, it may need a control response by an increase or decrease some parameters such as temperature or pressure. On the other hand, from energy concepts the delay and node degree are related to sensing and transmission to the data sink. There are few pieces of research which are focusing on real-time communication in MSSN.

Some of the researchers analyzed communication technologies. This is because wireless communication states consume high energy of sensor node when compared with stored sensing data. Additionally the receiver and transmit states of communications consume most of the energy compared with CPU processing.

*Misurata University, Libya (salem.sati@it.misuratau.edu.ly) 
To minimize or prevent energy consumption, many energy metrics were considered in the literature researches. However, most of these researches ignore the delay and social aspect of the sensor networks. The simplest routing algorithm for MSSN is Epidemic [4], this protocol based on whenever a sensor node storing a sensing message encounters with another sensor node, it transmits a replica of the sensed message. It forwards the message to the encountered node. This occurs when both nodes in coverage range. Moreover, there is a buffer space on the nodes which has no copy. However, such flooding-based and blind replica also causes relatively high energy consumption. To overcome the problems of Epidemic routing, many routing protocols limit the number of replicas, such as [5, 8]. Generally, the delivery ratio of flooding-based strategies is relevant high, but the heavy load of nodes may cause serious congestions or energy issues. To investigate how to control the forwarding list and how to order it there is some publications analysis this issue [7].

In our research paper, while the contribution of the paper presents a new opportunistic routing protocol based on Epidemic behavior. This new routing protocol can optimize energy consumption and delay in MSSN. This paper finds out a controlled threshold that can consider the requirements of social sensing, i.e. delay, energy and node degree as a control function for message replication. The following sections of this paper are organized as follows. Section 2, demonstrates the previous researches as related work. While section 3 provides the proposed routing protocol EERP and its model analysis. Section 4, the paper presents the updated version of EERP which named EERP + S. Simulation sets and performance metrics have been given in section 5. Simulations results and analysis are presented in section 6 . Finally, section 7 gives the conclusion and future work.

2. Related Work. There are many opportunistic routing that can be deployed in WSNs. One example of these routing is (OWR) suggested by [9]. This routing considered a practical scheme for WSNs. The idea of this protocol is inspired by the original ExOR [10] routing. ExOR designed for mesh networks. OWR protocol is implemented for a duty-cycled configuration. Moreover, data units are marked to sets of specific receivers. These receivers are forwarded by the next-hop that wakes up and successfully accepts the message. This behavior reduces the delay due to the source spent time for a specific node to wake up. Also, it reduces energy consumption for path transmission in the network. The energy reduction due to deploying all neighbors as critical forwarders.

Several routing protocols for WSN have been proposed. For example the paper [11] suggests an energy cost. This cost is optimally limited and increase the nodes lifetime. Also, the paper [12] has proposed a couple of energy concentrate data-forwarding rules for single path and multiple paths. The paper suggests a mechanism to minimize energy consumption via this cost. Moreover, this paper finds a trade-off between node energy and message delivery ratio. The paper [13] which takes into account the nodes residual energy as forwarding criteria. However, in all of the mentioned papers, the consideration of latency is missing and there are more wastage of message dissemination in wireless sensor networks. The main aim of opportunistic routing costs is the concept of minimizing the number of message copies to keep energy. Furthermore, applying the advantages of flooding is the nature of wireless networks. One advantage of flooding to send a message through multi-path in the network as possible. ETX was a first metric suggested for opportunistic routing especially in wireless networks. Researchers have implemented new routing costs and weights such as OEC (Opportunistic End-to-end Cost)[14], Also there is another research concerning Opportunistic Expected One hope Throughput (EOT)[15]. These two papers $[14,15]$ show the trade-off between the advancement of messages and the message forwarding time. Furthermore, the authors of the paper [16]suggest a Distance-based Energy Aware Routing (DEAR). DEAR ensures energy saving and balancing based on different energy and traffic analyzing models. Moreover, the paper [17] is address the power efficiency issue by suggesting a Real-Time routing with Controlled Dissemination (RTCD) of the message to mobile sink. RTCD consists of two stages which are flooding and routing stages. These two stages considered as data collection done by the mobile sink. This mobile sink reduces the energy consumption of the nodes. Controlling message dissemination is accomplished by limiting the topology diameter. In addition to setting a triggered value for the remaining energy of the sensors. Simulation results show that the delivery ratio for RTCD routing is better than other protocols. Finally, in this paper [18], authors suggest opportunistic routing protocol for sensor networks, This protocol based on the selection of the best forwarder node. This selection of neighbor node is the main factor that enhances the power prevention and nodes lifetime. To improve routing performance more efficiently, authors present a sleep algorithm named, PSS algorithm for the sensor 
nodes combined with opportunistic routing. Simulation results show that proposed routing has optimal energy consumption with less overhead.

3. Energy Efficient Routing Protocol (EERP). As the MSSN runs continuously such as real-time monitoring scenarios, the sensors will eventually die due to unnecessary message replication which leads to energy wastage. The problems raised by the energy limitation of mobile social sensors. There are mainly two aspects: (1) The message transmission which is the main factor of energy consumption; (2) The sensing data stored in the nodes which may be delayed as real-time application. The first point will impact immediately on sensors' energy consumption of the network, it directly degrades the routing performance from the reliability concept. The second point will lead to poorly end-to-end delay of the real-time application in MSSN, this will additionally effect on the energy of the sensors where stored message also will consume a piece of power. From the perspective of energy saving and real-time decision, this paper presents an Energy-Efficient Routing Protocol(EERP) for MSSNs. The basic idea as follow: To reduce the complete energy loss of sensor node we propose a new scheduling mechanism. This scheduling strategy derives the optimal number of message replication according to variables. These variables are the transmission and the number of stored sensing data. Moreover, both variables impact on total energy consumption.

The number of messages generated by traditional mobile social routing tends to be large, resulting in an insufficient energy consumption (i.e., the energy consumption of flooding routing in social sensing topology is often much larger than that in fixed WSN.). To overcome this challenge, based on the opportunistic scheduling, the message life is considered as the main factor in the energy. The messages are ordered in the scheduling queue of the node's buffer. Then in the forwarding decision phase, we implement the energy ratio of the message balance. Moreover, the energy ratio depends on the number of transmitted and stored copies. This forwarding strategy of EERP improves the ROUTING performance of distributed and cluster topologies. In terms of optimal message replication, we take the life of the message into account as a part of the residual energy. This is basically different from traditional social sensing protocols which consider only the social tie. The main advantages points of proposed routing protocol include the following:

- The optimal threshold of replication is derived to minimize the sensors' energy consumption of MSSN.

- Message transmission and sensing are both considered as the energy balance parameters.

- The message life energy ratio is necessary to compute the energy consumption instead of a social tie only in MSSN.

- A new forwarding priority function can ensure the message with lower energy loss.

- Sensor node residual energy can be saved when deploying the proposed forwarding function.

- The new routing achieves better routing performance by reduction of storage, energy, and delay.

The aim of EERP is to minimize the energy consumption of the network by limiting the number of message. Recall that in traditional social networks the message will not be replicated to the met node always, but message replicated when the node which holds the message has lower social weight. Here, Social measuring could be any existing social indicators, such as node degree or centrality.

EERP is a routing protocol to reduce the traffic load of each node. It can be deployed to any social-based distributed or clustered topology. EERP makes its decision using energy and sensing factors. Traditional social routing uses social metrics per node for its ordering and forwarding decisions. Where to compute the social weight of a node, a social graph is required to express the social ties among all nodes. Commonly such a social graph is built from recorded contacts of the node with other nodes in the network. Assume that $\mathrm{N}$ is the set of nodes in the social network. Each sensor node can transmit and receive messages when it connected with another node. The message replicated when other node has higher weight and has no copy.

The EERP uses a threshold on queue order to satisfy whether there is a chance between two messages to be replicated. If the number of transmission and stored times between the two messages is equal. Then there is an energy balance between these two messages based on their life TTL.

In traditional social routing protocols, the messages are replicated to the contacted nodes which have a higher social weight. This may grantee to achieve best delivery ratios, but nodes that have large social wight may die soon due to their huge traffic load. Therefore, we consider other parameters in proposed EERP compared with traditional social-based sensing routing in MSSN.

EERP decision is based on the energy metric of message and node. These energy metrics are transmission 
and storing times of the message copy. Here, energy ratio $0 \leq E_{\%} \leq 1$. Thus, it becomes more difficult for the current message to transfer. This is because the other message needs to have energy ratio less than that of the message to be forwarded. By applying this rule, the number of message redundancy in the network will be minimized. Naturally, the delivery of the new replication decision decreases, thus we dynamically modified the energy function based on the Time To Live (TTL). The dynamic behavior of the decision is to minimize the energy consumption. TTL of the message shows whether the message is expired and when the message should be evicted. At the message originator, the TTL of a message is configured to an initial value $T T L_{0}$. After each transmission or forwarding decision, the value of TTL will be copied to the new copy. When TTL is reached to zero, the message will be expired and evicted from the node's buffer.

EERP forwarding decision is made only when the energy function of the message is less than that of the message to be forwarded. The basic concept of the dynamical energy function ratio is as follows. In the beginning, when the message created TTL is set to maximum, EERP puts minimizing the energy ratio of the message as its first priority, thus the value of energy ratio subtracted from 1 . However, after several transmission and storage times, when TTL goes to a small value, which leads to that message will be evicted soon, EERP modifies the energy ratio by considering the transmission and sensing times as its first priority. Therefore, we set the energy ratio as:

$$
F_{d}=M S_{\left(S_{e}, T_{r}\right)} \cdot E_{\%}
$$

where $f_{d}$ is a forwarding decision function which consists of two parts used to determine the threshold of message replication, The first part $M S_{\left(S_{e}, T_{r}\right)}$ is related to mobile sensing where data sink and sensors considered as mobile devices. The second part $E_{\%}$ related to the energy ratio of the message. The following equation shows the factors which impact on the forwarding decision as follow:

$$
F_{d}=\left(1+T_{m}+S_{m}\right) \cdot\left(1-\frac{T T L_{r}}{T T L_{0}}\right)
$$

The mobile sensing function considers the two main function of the sensing. These functions are transmission $T_{m}$ and sensing $S_{m}$. On the other hand, the message energy ratio based on $T T L_{0}$ and $T T L_{r}$ are the initial message TTL value and the current TTL value of the message, respectively. Note that EERP considered as mobile sensing energy aware routing protocol when deploying the forwarding function. Algorithm 1 shows the detailed description of the proposed EERP.

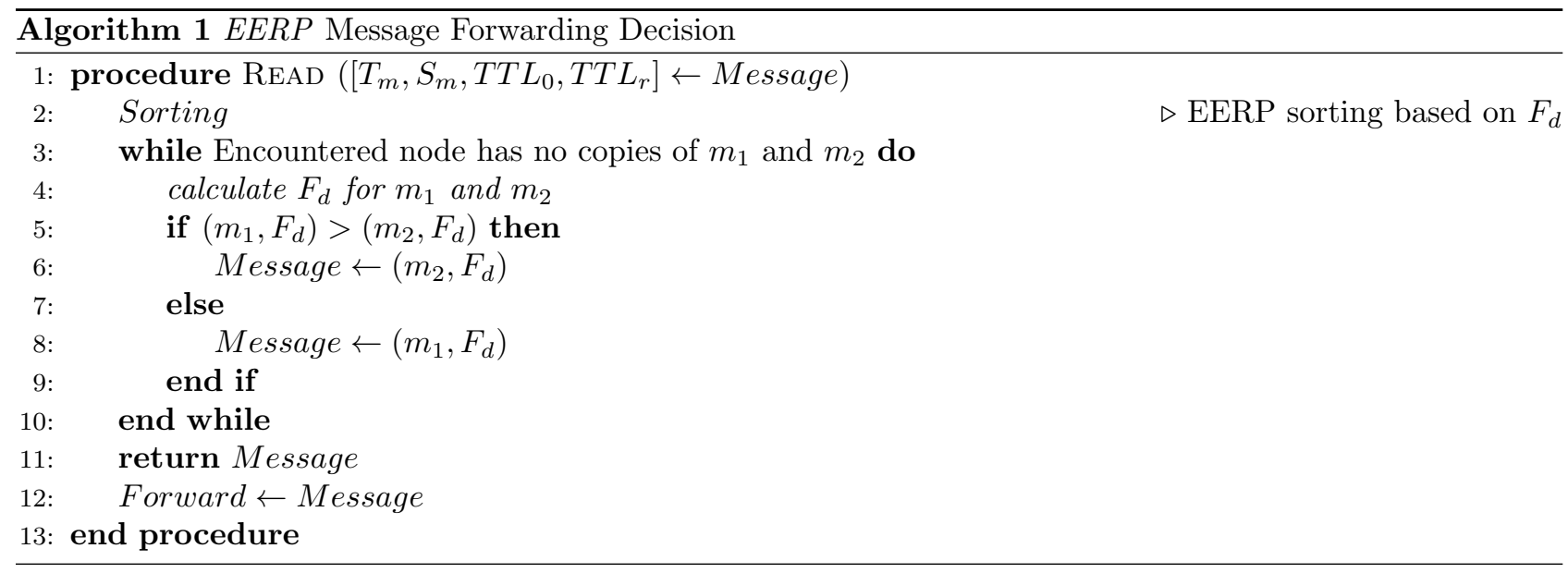

4. Enhanced Energy Efficient Routing Protocol $($ EERP+S). We improve the proposed EERP by considering the social metric. This social metric related to the energy of the node instead of the message only as in EERP. Where the message replicated to a specific group of connected neighbors. The traditional replication is based on replication to any number of neighbors. The new strategy reduces the energy consumption of the 
node. Energy-saving achieved by replicating the message to multiple nodes with single message energy $T_{m}$. We adopted the forwarding decision of EERP $+\mathrm{S}$ by taking into account the social metric. EERP $+\mathrm{S}$ combines the forwarding function of EERP with the energy consumption of the node. This node energy saving is based on a social metric which is node degree $N_{d}$. Where it might be better to consider message and node energy. As a social metric, it is more efficient to find a node that its activity is larger than other nodes. This phenomenon could have the best impact on energy consumption. To improve the forwarding decision of EEPR+S, we include an additional node energy metric $\left(N_{d}\right)$. This metric is used to further dynamically adjust energy consumption based on EERP. When a node encounters more than a specific number $N_{d}$ of nodes that have no copy of the message, it forwards the message to all $N_{d}$ or greater number of nodes. When the number of nodes is less than $N_{d}$, it does not forward the message (since the activity of the met nodes is not better than the current node). Our proposed enhanced protocol EERP $+\mathrm{S}$ slowly relaxes the forwarding decision by combining the message and node energy consumption indicators. In this improved version EERP+S, the forwarding function is dynamically adjusted by both $F_{d}$ of the message and the number of met nodes (i.e., node degree). These nodes buffers have no copy of the message. Here, $N_{d}$ is threshold calculated depending on the node degree. This node degree is related to the radio range of the sensor node interface as shown in the following equation:

$$
(E E R P+S)= \begin{cases}E E R P & \left(F_{d}\right)_{\text {message }}<\left(F_{d}\right)_{\text {others }} \\ N_{d} & N_{d} \geq \sqrt{r}\end{cases}
$$

5. Simulation Scenarios. This section conducts simulation experiments over social scenarios. The comparison is evaluated two proposed EERP and EERP+S with Epidemic and its social version protocols. The evaluation with other protocols conducted using ONE simulator[6]. The evaluation uses the EERP based on $F_{d}$ as a routing protocol for MSSN. Additionally, it deploys the enhanced protocol EERP+S by using the node degree as the social metric. Obviously, in EERP $+\mathrm{S}$ routing, the message has more chance of forwarding to the met node. The forwarding decision satisfied when two conditions are available. First. the node has a high social metric. Second, the message has low energy consumption.

The EERP is an energy-weighted protocol. We compare EERP and EERP $+\mathrm{S}$ with the traditional following existing routing protocols.

1. Epidemic: When the node connected with any encountered neighbor nodes, the node which holds the message copy it to any number of neighbors nodes.

2. Ep-Soc: The message is only forwarded from the node which holds the message to the connected nodes. The forwarding decision is true when the number of neighbors is equal to or greater than $N_{d}$. The value of $N_{d}$ calculated based on $N_{d}=\sqrt{\text { Radio Range }}$.

Our simulation scenario is a set of parameters for sensing environment such as pollution. This use case simulated by the ONE simulator. These settings are used for different routing protocols. This paper evaluates only the sensing and transmission of data.

The message TTL and sensing interval are changed for simulate MSSN scenario. The evaluation is to address various kinds of social sensing use cases. Table 5.1 shows the basic settings for MSSN scenarios, they are derived from the social settings from the ONE simulator. The simulations of different routing protocols run for 720 minutes. It simulates 125 sensor nodes.

Nodes generally divided into groups. The nodes have a buffer space limited by the number of messages. Sensor nodes can only move on different routes, which simulate streets and buildings in the smart city. There are two types of sensors, which are carried by humans as smart-phones or fixed on cars. In addition. We simulate 5 mobile data sinks, these data sinks have a large memory space compared with other sensors nodes. In all our simulation experiments, we evaluate the performance of different routing protocols using the following metrics.

1. Data Delivery Ratio: the average ratio of the successfully delivered sensed data from the sensors to the mobile data sinks.

2. Overhead Percentage: the number of relayed sensed data to the total successfully delivered data to mobile data sink.

3. Average Delay: the average time spent to successfully delivered sensed data from the sensors to the mobile data sink. 
TABLE 5.1

Simulation Settings

\begin{tabular}{|l|l|l|}
\hline No & Settings & Value $(\mathrm{s})$ \\
\hline 1 & Simulation Region & 4500 in $3500 \mathrm{~m}^{2}$ \\
\hline 2 & Simulation Duration & $720 \mathrm{~min}$ \\
\hline 3 & Number of nodes & 125 \\
\hline 4 & Sensors Type with speed & $\begin{array}{l}80 \text { Smart-phones Sensors }(0.5-1.3 \mathrm{~km} / \mathrm{h}) \\
40 \text { Vehicular Sensors }(10-75 \mathrm{~km} / \mathrm{h}) \\
5 \text { Mobile Data Sink }(15-60 \mathrm{~km} / \mathrm{h})\end{array}$ \\
\hline 5 & Routing protocols & $E E R P, E E R P+S$, Epidemic and Ep-Soc \\
\hline 6 & NIC & One-to-All \\
\hline 7 & Radio Range & $0.25 \mathrm{~km}$ \\
\hline 8 & Link Speed & $0.25 \mathrm{MBps}$ \\
\hline 9 & Social Metric & Node Degree \\
\hline 10 & Sensing Data Generated & $500-1024 \mathrm{~KB}$ \\
\hline 11 & Sensing interval & $25-35 \mathrm{~s}$ \\
\hline 12 & Sensing Data (TTL) & $120,240,360,480,600 \mathrm{~min}$ \\
\hline 13 & Sensor Memory Space & $\begin{array}{l}\text { Smart-phones: } 5 \mathrm{MB} \\
\text { Mobile Data Sink: } 50 \mathrm{MB}\end{array}$ \\
\hline
\end{tabular}

4. Relayed Messages: the average number of forwarding times needed to deliver a single successfully message.

5. Average Hop Counts: the average number of relayed nodes during each successful path.

6. Average Sensing Time: the average time spent in sensor memory during the delivery of the message.

6. Numerical Results. The evaluation of proposed EERP and EERP $+\mathrm{S}$ with traditional Epidemic and Ep-Soc routing as described in Table 5.1. Through our simulation social scenarios, we modify sensing data TTL from 120 to 600 minutes with a step of 120 minutes. This is for simulating different sensed data. Also, we pay attention to energy and social balancing for EERP forwarding decisions. In addition, we apply social metrics as additional criteria in the EERP $+\mathrm{S}$ routing protocol. We apply node degree $N_{d}$ as a threshold which computed based on radio range (r) and node activity. Both conditions of EERP and EERP $+\mathrm{S}$ depends on Eq. (3.2). For the EERP protocol scenario, we select the forwarding function of the message based on the energy threshold of $E_{\%}$. Also, we select the number of neighbors as the second tie for a proposed energy-efficient social sensing $\mathrm{EERP}+\mathrm{S}$.

6.1. Data Delivery Ratio. The data delivery ratio of EERP is compared with EERP $+\mathrm{S}$ which use scheduling based on $N_{d}$ in addition to $F_{d}$. We consider FIFO evict policy as default for all different routing protocols. Figure 6.1 shows the data delivery ratio of different protocols. The figure shows that EERP $+\mathrm{S}$ is better than Ep-Soc. This clear when deploying forwarding threshold based on social metric as node degree. The enhancement in data delivery ratio of EERP $+\mathrm{S}$ reaches $15 \%$ when compare with EERP by applying $F_{d}$ decision. Also it is better more than $3 \%$ when compare EERP $+\mathrm{S}$ with Ep-Soc at data sensed of TTL=600 min. The EERP $+\mathrm{S}$ enhancement due to combining of energy model of EERP with social function of node degree $N_{d}$. The social metric doesn't considered by EERP. EERP forwarding function has better performance when compared with traditional Epidemic. Obviously, the performance of EERP $+\mathrm{S}$ is the best because it minimizes the energy consumption of the data sensing. EERP when applies forwarding function $F_{d}$ considered as better than traditional Epidemic $(\approx 1-18 \%)$.

6.2. Overhead Percentage. The overhead percentage metric is considered as main energy consumption indication. Where it shows how much energy consumed for delivering the sensed data in distributed MSSN. Also the overhead percentage can be calculating by $N_{d}$ and $F_{d}$, where EERP $+\mathrm{S}$ eliminates more of overhead of EERP as shown in Figure 6.2. Clearly, traditional Epidemic and Ep-Soc are suffering from consuming of the total energy by applying unlimited and social forwarding decision respectively. Figure 6.2 shows that EERP $+\mathrm{S}$ 


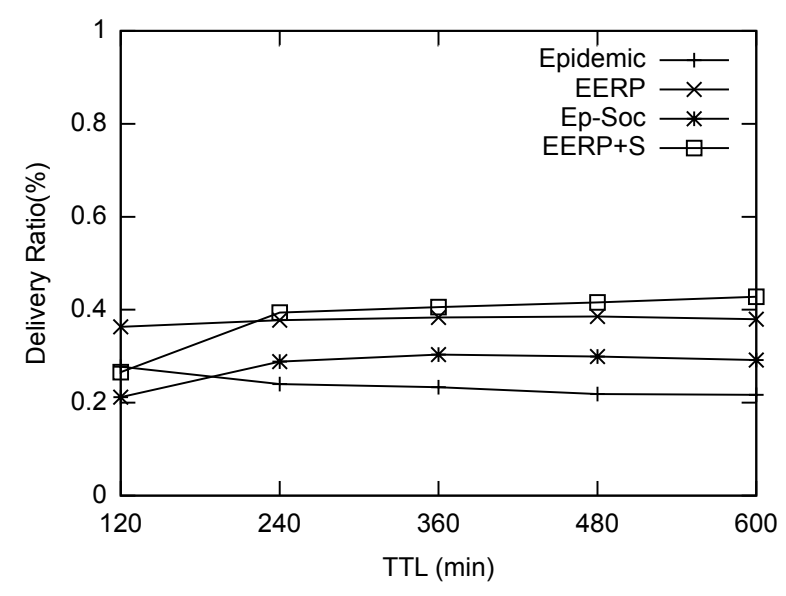

FIG. 6.1. Data Delivery Ratio

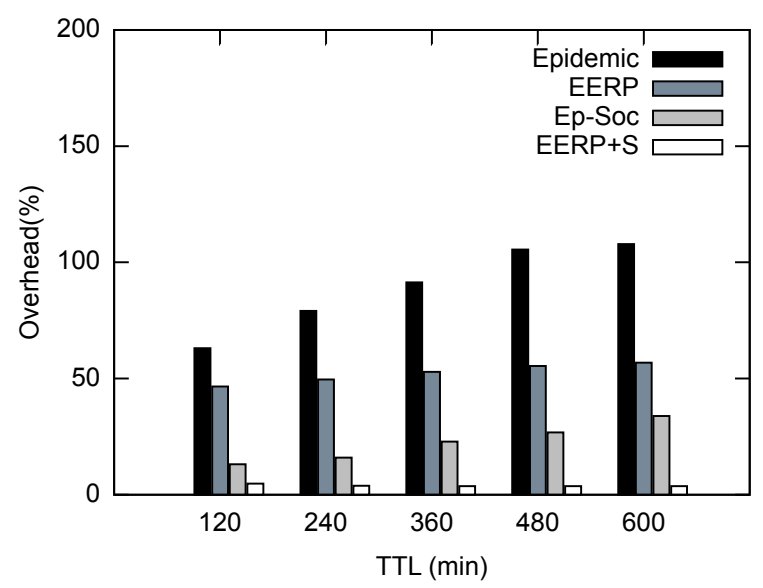

Fig. 6.2. Overhead Percentage

has lower overhead when combining $F_{d}$ and $N_{d}$ by deploying Eq. (4.1). EERP + S protocol minimizes the number of relayed messages percentage to $(\leq 10 \%)$. This is because the replication of social sensed data implemented based on energy percentage and node social metric. Furthermore, from figure 6.2, we observe that Ep-Soc has a regular overhead percentage $\leq 55 \%$ and EERP has $\leq 60 \%$ compared with traditional flooding Epidemic (60-110\%). The optimal threshold of the applied forwarding decision for EERP $+\mathrm{S}$ and EERP depends on node and message energy consumption, respectively. Where the main concept of forwarding decision of both EERP and EERP $+\mathrm{S}$ based on balanced between energy and message delivery.

Obviously, as we varying the TTL of sensed data for varying traffic load, in figure 6.2 the overhead percentage increases clearly when applying traditional Epidemic and quite when deploying Ep-Soc. This is because the social metric of node degree increase the delivered data through a high activity mobile nodes. Furthermore, figure 6.2 shows that EERP $+\mathrm{S}$ which deploy energy and social formulation still has lower overhead percentage $(\leq 10 \%)$. Furthermore, EERP $+\mathrm{S}$ has minimum overhead when compared with Ep-Soc and EERP.

6.3. Delay. The value of delay considered as application quality indication and main factor for quality of services (QoS). Commonly, delay is used as performance indication specially for real-time applications. Figure 6.3 shows that the lowest delay for different four protocols can be achieved when applying EERP, where EERP consider the optimal forwarding decision by $F_{d}$ as function of message energy. Basically, we found that the number of buffered times of sensed data is impact directly on total end to end delay. As one contribution, we get from delay metric results that the social metric is directly impact on delay of sensed data specially in 


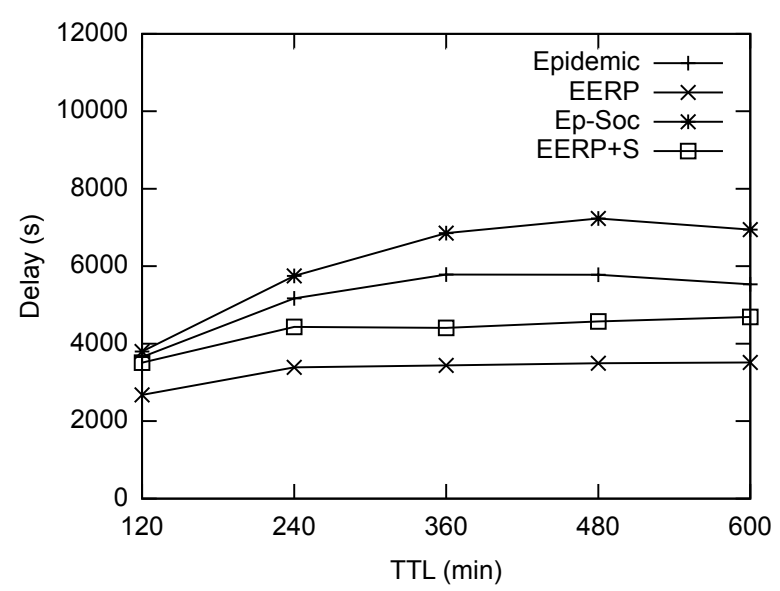

Fig. 6.3. Delay

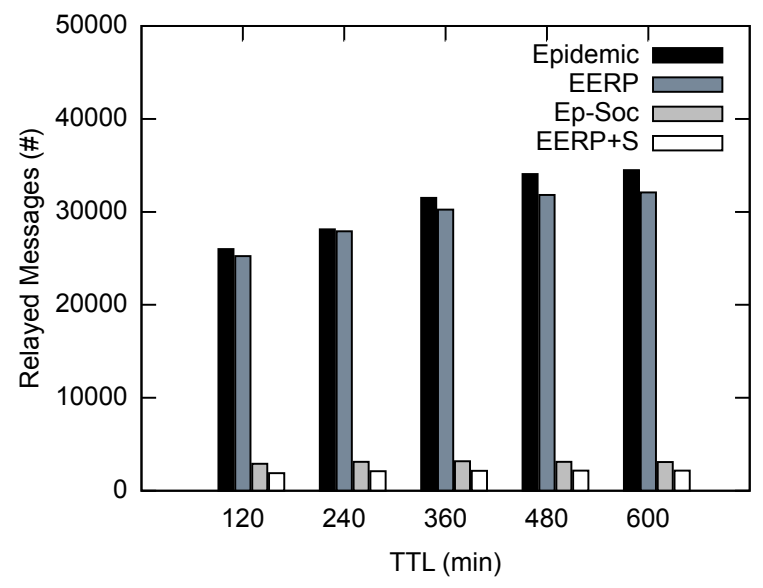

FiG. 6.4. Relayed Messages

mobile social sensing networks. The forwarding decision formulation which used by EERP will impact on the average sensed data delay. As second advantage, it also minimize the energy when considering radio range as degree variable of EERP $+\mathrm{S}$. Therefore, $\mathrm{EERP}+\mathrm{S}$ considers the social and energy as criteria of forwarding functions. Moreover, we observe that when we apply different forwarding based on each routing deployed as shown in Figure 6.3 , EERP has minimum delay $(\approx 3000 s$ at TTL $=480 \mathrm{~min})$ when it compared with EERP $+\mathrm{S}$ and Epidemic $(\approx 4500 s$ and $\approx 5800 s$,respectively). Additionally. social epidemic Ep-Soc has a higher delay ( $\approx 7000 \mathrm{~s})$. Furthermore, Figure 6.3 demonstrates that EERP has minimum data sensed delay when it deploy its forwarding decision by applying Eq. (3.2). This can be observed specially when compare EERP with EERP+S by deploying Eq. (4.1) as forwarding decision which has social metric as second tie.

6.4. Relayed Message. The energy consumption in MSSN is very criteria. Mobile sensors have limited energy. Therefore, it is better to design routing protocol which decrease the number of relayed sensed data. As the number of relayed messages is decreased as the energy efficacy is increased, but at the same time, routing protocol should keep the desired data delivery percentage or it is better to increase it. The main concept of EERP and EERP $+\mathrm{S}$ are optimize the number of sensed data with keeping of desired delivery percentage. Relayed sensed data as energy indication is demonstrated by figure 6.4. This figure shows relayed messages of EERP and EERP+S in comparison with Ep-Soc and traditional Epidemic.

In addition, the number of relayed messages sensed is calculated for every routing protocol. Proposed protocols are apply different forwarding decision of EERP and EERP $+\mathrm{S}$. Basically, EERP aims to reduce 


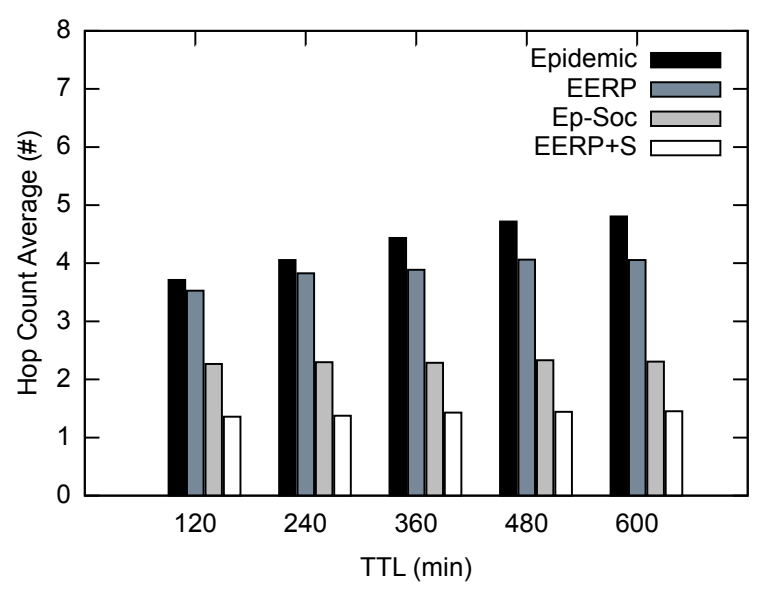

FIG. 6.5. Hop Count Average

energy consuming of sensors nodes by calculating energy percentage as TTL function.

Figure 6.4 shows the results of energy and social consideration of EERP and EERP + S, respectively. Figure 6.4 shows that Epidemic relays $\approx 33000$ more data than EERP which exchanges $\approx 31000$ messages at TTL $=480 \mathrm{~min}$. Also Ep-Soc relays $\approx 2200$ and EERP $+\mathrm{S}$ relays only $\approx 1800$ sensed data at TTL $=480 \mathrm{~min}$. We observe from figure 6.4 that the two social protocols EERP $+\mathrm{S}$ and Ep-Soc have minimum relayed messages. Furthermore, they are lead to more delay compared with other protocols as figure 6.3 demonstrated. EERP when deploys the function of $F_{d}$ it relays $\approx 23000$ sensed data at TTL $=120$ min and $\approx 31000$ sensed data at $\mathrm{TTL}=600 \mathrm{~min}$. This due to that EERP reduces the number of sensed data to $\approx 20 \%$. EERP when compared with traditional Epidemic at TTL $=600$ Epidemic relays $\approx 33000$ sensed data. Epidemic is the highest values for relayed messages in figure 6.4. This is due to it doesn't consider any energy or social metrics. Figure 6.4 demonstrates that EERP $+\mathrm{S}$ eliminates $\approx 5 \%$ of sensed data when compared with Ep-Soc at different TTL values. This due to the fact that $\mathrm{EERP}+\mathrm{S}$ is also consider the EERP forwarding decision instead of social metric only. This advantage of energy percentage is to enhance the EERP $+\mathrm{S}$ and EERP compared with Ep-Soc and Epidemic respectively.

6.5. Hop Count Average. Hop count is one of energy and social metric. Concerning energy it indicates that the number of times the energy consumed during transmission and receiving, in addition it shows the sensing time of sensed data. On the other hand hop count as social metric. It shows the social tie of the nodes in the path with message destination. As the number of hops increases as the social metric is decreases between the source and destination. Basically, when the hop count decreases then the energy consumption decreases and social tie increases. For our evaluation experiments, we consider the hop count average to compare EERP and $\mathrm{EERP}+\mathrm{S}$ with other competitive protocols. It is important to take into account the hop count as metric for social and energy costs. This helps on adapting between sensing time and energy consumption of the data during the same path. When the hop count is averaged for all successfully received sensed data. It shows the average energy consumption between sensor node and data sink.

Figure 6.5 shows the results of different applied routing protocols for comparison based on average hop count. Figure 6.5 demonstrates that EERP + S has lowest average hop count $<2$. Ep-Soc has average hop count $>2$ which means that EERP + S perform better than Ep-Soc. When apply the forwarding decision of EERP, it has average hop count $\leq 4$ when compared with traditional Epidemic which has average of (5-6). As the hop count increases as the message consumes more energy, In addition, the node consumes more resources such as bandwidth and memory. On the other hand, as the hop count average decreases it ensures that both congestion and energy will decrease. We observe from figure 6.5 that when deploying EERP+S the hop count for all paths will reduced to $\approx 40 \%$. This percentage of EERP+S hop count reduction is when compared with traditional Epidemic. 


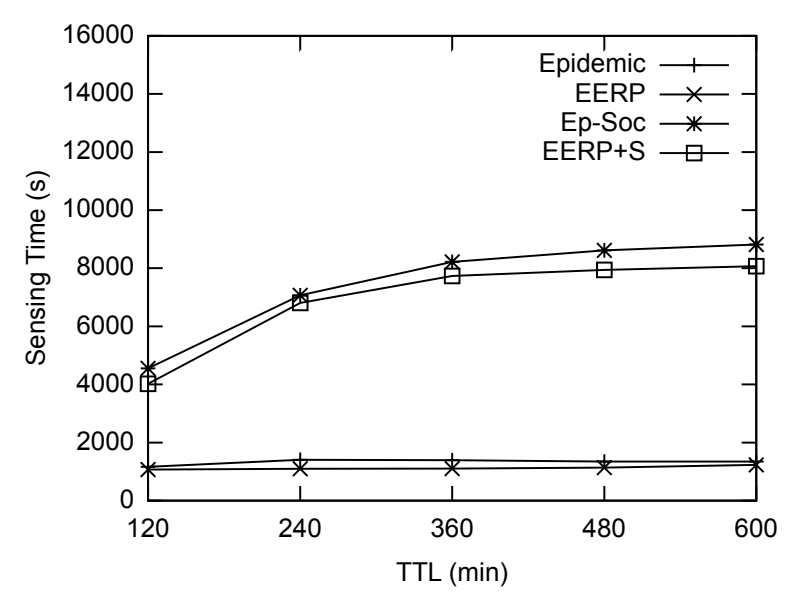

FIG. 6.6. Sensing Time

6.6. Sensing Time. The sensing time is the time spent on the sensor nodes buffers. This time is spent by sensed data till reach the mobile data sink. For detailed analyze of sensed data, simulation scenarios consider the sensing time as indication of real-time delay. We apply $F_{d}$ in both proposed protocols for minimize the energy consumption of message and the node. We analyze EERP and EERP $+\mathrm{S}$ which both consider the energy percentage by deploying message TTL. Figure 6.6 shows the that traditional Epidemic reaches sensing time higher than EERP, where EERP $+\mathrm{S}$ reaches minimum sensed data time when compared with Ep-Soc. EERP $+\mathrm{S}$ sensing time reaches higher values when compared with EERP. This insufficient sensing time of EERP $+\mathrm{S}$ caused by social tie of EERP+S.

$\mathrm{EERP}+\mathrm{S}$ has a routing metric which based on the energy percentage and social tie of node degree. Figure 6.6 shows that as TTL increases as the sensing time increases. Sensing time is increased due to as TTL increases the congestion will normally increases.

The sensed time, i.e. buffering delay, is one of the component of average delay in addition to the transmission delay. This time has great impact on the energy consumption of the node.

$\mathrm{SSRP}+\mathrm{S}$ increase sensing time because it minimize the number of paths for the sensed data. But EERP increases the number of sensed data paths which minimize this time. On the other hand, multi path has a disadvantage from the view of the energy, where multi path increases the overhead percentage.

Essentially, the routing protocol's forwarding decision may considered one of factors which leads to increase the energy consumption. On the other hand, limiting or reducing the number of sensed data copies minimizes the energy consumption. In addition it increase congestion which leads to increasing the average sensed time. Therefore, we compare EERP with traditional Epidemic by taking into account the forwarding decision based on energy percentage. As shown in figure 6.6, we apply different forwarding function depending on every routing protocol. EERP has a minimum sensed time $(\approx 1100 \mathrm{~s}$ at $\mathrm{TTL}=360 \mathrm{~min})$ when it compared with traditional Epidemic which has sensing time of $(\approx 1700 s)$. In addition to social routers EERP $+\mathrm{S}$ and Ep-Soc which have sensing time of $(\approx 7800 \mathrm{~s}$ and $\approx 8100 \mathrm{~s}$, respectively $)$ at $\mathrm{TTL}=360 \mathrm{~min}$.

7. Conclusion. Mobile social sensing routing is very important for sensor networks. The nodes in MSSN are suffering from limited resources such as energy. This paper considers forwarding straggly for MSSN routing. The paper investigates the flooding or blind forwarding strategies, these forwarding strategies are commonly used by an Epidemic with or without social metric (Ep-Soc). These protocols are target to achieve a high performance without considering the delay and energy in MSSN. We inspired by the two Epidemic routing versions (Ep-Soc \& Epidemic) two suggested routing protocols (EERP+S \& EERP). The two suggested protocols are with and without social metric of node degree. In addition, we consider the criteria of energy as main factor of our design for both protocols. Our simulation evaluation was in terms of data delivery ratio, delay and overhead percentage. Based on energy design, we implement social version EERP $+\mathrm{S}$ by deploying the social metric of node degree. We formulate the forwarding decision of EERP based on energy percentage, where enhanced 
version $\mathrm{EERP}+\mathrm{S}$ considered as energy-based and social-based routing protocol. Simulation results using ONE simulator are analyzed to evaluate proposed protocols. Our proposed protocols improves the data delivery ratio. Moreover, both protocols are decrease the overhead and relayed messages. Additionally, the proposed routing protocols decrease average delay and sensing time. EERP and EERP $+\mathrm{S}$ are reduce the average hop count. We observe this when compare EERP \& EERP $+\mathrm{S}$ with Epidemic \& Ep-Soc protocols. Therefore, combining social features with energy aware forwarding techniques are significant for improve MSSN routing. These proposed protocols are an efficient routing protocols for MSSN. As future work, we have planning to consider the number of nodes as density parameter. We will investigate node density impact on the proposed routing protocols.

\section{REFERENCES}

[1] B. Hull, V. Bychkovsky, K. Chen, M. Goraczko, A. Miu, E. Shih, Y. Zhang, H. Balakrishnan, and S. Madden, CarTel: A Distributed Mobile Sensor Computing System, ACM SenSys, (2006), pp. 125-138.

[2] B. Kranstauber, A. Cameron, R. Weinzerl, T. Fountain, S. Tilak, M. Wikelski, and R. Kays, The Movebank data model for animal tracking, Journal of Environmental Modelling and Software, Vol.26. No.6 (2011), pp. 834-835.

[3] L. Ferrari, and M. MameI, Discovering daily routines from Google Latitude with topic models,International Conference on Pervasive Computing and Communications, PerCom 2011, 21-25 March 2011, Seattle, WA, USA, Workshop Proceedings, pp. $432-437$.

[4] A. Vahdat and D. Becker, Epidemic Routing for Partially-Connected Ad Hoc Networks, Technique Report, Department of Computer Science, Duke University, USA, vol.20, no. 6, 2000.

[5] S. SATi, A. IpPisch, AND K. GRAFFi, Dynamic replication control strategy for opportunistic networks, in 2017 International Conference on Computing, Networking and Communications, ICNC 2017, Silicon Valley, CA, USA, January 26-29, 2017, 2017, pp. 1017-1023.

[6] A. Keränen, J. Ott, And T. KärkkÄInen, The ONE simulator for DTN protocol evaluation, Proceedings of the 2nd International Conference on Simulation Tools and Techniques for Communications, Networks and Systems, SimuTools 2009, Rome, Italy, March 2-6, 2009, pp. 55.

[7] S. Sati, C. Probst, and K. Graffi, Analysis of Buffer Management Policies for Opportunistic Networks,in 25th International Conference on Computer Communication and Networks, ICCCN 2016, Waikoloa, HI, USA, August 1-4, 2016, pp. $1-8$.

[8] S. Sati, A. Sohoub, A. Eltahar,K. Bin Ahmad, K. Ahmad,and A. Bakeer, Degree Contact DC-Epidemic Routing Protocol For Opportunistic Networks, Advances in Wireless and Optical Communications (RTUWO) 2018, Riga, Latvia, November $15-16,, 2018$, pp. 198-203.

[9] O. Landsiedel, E. Ghadimi, S. Duguennoy, and M. Johansson, Low power, low delay: opportunistic routing meets duty cycling, The 11th International Conference on Information Processing in Sensor Networks (co-located with CPS Week 2012), IPSN 2012, Beijing, China, April 16-19, pp. 185-196.

[10] S. BISWAS, AND R. MORRIS, Exor: opportunistic multi-hop routing for wireless networks ,SIGCOMM Computer Communication Review, vol. 35, no. 4,pp. 133-144.

[11] Q. Cao, T. He, L. Fang, T. Abdelzaher,J. Stankovic, and S. Son, Efficiency Centric Communication Model for Wireless Sensor Networks, Proceedings of 25th IEEE INFOCOM, Barcelona, Spain. 2006, pp. 1-12.

[12] M. Busse, T. Haenselmann, And W. Effelsberg, An Energy- Efficient Forwarding Scheme for Wireless Sensor Networks, Proceedings of WOWMOM06, IEEE Computer Society, Washington, DC, USA. 2005, pp. 125-133.

[13] M. Busse, T. Haenselmann, and W. Effelsberg, Poster-Abstract: A Lifetime-Efficient Forwarding Strategy for Wireless Sensor Networks, Wireless Sensor Network. [Poster Abstract], 2006; 20.

[14] M. Hung, K. Lin, C. Hsu, C. Chou,ANd J. Tu, On enhancing network-lifetime using opportunistic routing in wireless sensor networks, Proceedings of 19th International Conference on Computer Communications and Networks (ICCCN), Zurich. 2010, pp. 1-6.

[15] C. Hsu, H. Liu, and W. Seah, Opportunistic routing - A review and the challenges ahead ,Computer Networks. 2011; 55(15), pp. 3592-3603.

[16] J. Wang,J. KIm, L. Shu,Y. NiU, ANd S. LeE, A distance-based energy aware routing algorithm for wireless sensor networks, Sensors. 2010; 10(10):,pp. 9493-9511.

[17] R. Mikkili, and J. Thyagarajan, A real-time routing protocol with controlled dissemination of data queries by mobile sink in wireless sensor networks, Indian Journal of Science and Technology. 2015 Aug; 8(19),pp. 1-10.

[18] S. BABA, AND K. RaO, Improving the Network Life Time of a Wireless Sensor Network using the Integration of Progressive Sleep Scheduling Algorithm with Opportunistic Routing Protocol, Indian Journal of Science and Technology. 2016 May; 9(17),pp. 1-6.

Edited by: Ashish Kr. Luhach

Received: Jun 26, 2019

Accepted: Sep 26, 2019 